\title{
Intraortic Pulse Pressure Is Correlated With Coronary Artery Stenosis
}

\author{
Morihiro OZAKI, ${ }^{1} \mathrm{MD}$, Hiroshi MASUOKA,${ }^{2} \mathrm{MD}$, Atsushi KAWASAKI, ${ }^{3} \mathrm{MD}$, \\ Masaaki ITO, ${ }^{4} \mathrm{MD}$, and Takeshi NAKANO,${ }^{4} \mathrm{MD}$
}

\begin{abstract}
SUMMARY
There is increasing evidence that peripheral pulse pressure measured at the brachial artery is a good predictor of coronary heart disease. However, the relation between pulse pressure and angiographically demonstrated coronary artery stenosis has not been fully elucidated. We designed the present study to investigate the association of the various components of blood pressure, such as systolic pressure, diastolic pressure, and pulse pressure of both peripheral and central arteries with angiographically determined coronary artery stenosis. Levels of aortic systolic pressure, aortic diastolic pressure, aortic pulse pressure, peripheral systolic pressure, peripheral diastolic pressure, and peripheral pulse pressure were determined in 323 patients who underwent diagnostic coronary angiography. Of these 323 patients, 215 patients had significant organic coronary artery stenosis. Aortic pulse pressure was significantly higher in patients with coronary artery stenosis $(P$ $=0.0050$ ). Aortic diastolic pressure was lower in patients with coronary artery stenosis (marginally significant, $P=0.0462$ ). However, no statistically significant difference was observed between other blood pressure components and coronary artery stenosis. Multivariate analyses showed that aortic pulse pressure was associated with coronary artery stenosis independently of aortic diastolic pressure. Moreover, aortic pulse pressure was positively correlated with the number of vessels involved $(P=0.0024)$. The results of the present study indicate that aortic pulse pressure is significantly and independently correlated with angiographically determined coronary artery stenosis. (Int Heart J 2005; 1: 6978)
\end{abstract}

Key words: Pulse pressure, Coronary artery stenosis, Aortic pressure

SINCE the introduction of the mercury-column sphygmomanometer at the beginning of the 20th century, a number of epidemiological studies have shown a close relation between the level of peripheral arterial pressure and coronary heart disease. ${ }^{1-6)}$ Currently, there is increasing evidence that peripheral pulse pressure measured at the brachial artery using the cuff method is a good predictor of coro-

From the ${ }^{1}$ Division of Internal Medicine, Yokkaichi Social Insurance Hospital, ${ }^{2}$ Division of Cardiology, Ueno Municipal General Hospital, ${ }^{3}$ Division of Cardiology, National Mie Central Hospital, and ${ }^{4}$ First Department of Internal Medicine, Mie University School of Medicine, Mie, Japan.

This work was supported in part by a Grant-in-Aid (2001) from the Mie Medical Research Foundation.

Address for correspondence: Morihiro Ozaki, MD, Division of Internal Medicine, Yokkaichi Social Insurance Hospital, 10-

8 Hazuyama-chou, Yokkaichi, Mie 510-0016, Japan.

Received for publication January 6, 2004.

Revised and accepted July 22, 2004. 
nary artery disease events. ${ }^{5-9)}$ In 1997 , Benetos, et al reported a series of 19,083 French men followed for an average of 19.5 years. ${ }^{8)}$ This study showed that a wide brachial pulse pressure is a significant independent predictor of all-cause, cardiovascular, and especially, coronary mortality. In 2001, Franklin, et al reported a series of 3060 men and 3479 women who were enrolled in the Framingham Heart Study and followed for an average of 17 years. ${ }^{\text {) }}$ They reported that brachial pulse pressure was the strongest predictor of coronary heart disease in the patients greater than 60 years of age. However, the relation of pulse pressure with angiographically demonstrated coronary artery stenosis has not been fully elucidated. The purpose of this study was to investigate the association of the various components of blood pressure, such as systolic pressure, diastolic pressure, mean pressure, and pulse pressure of both peripheral and central arteries with angiographically determined coronary artery stenosis.

\section{METHODS}

Study population: The study population comprised 416 consecutive patients who underwent diagnostic coronary angiography for possible coronary artery disease. Patients who had liver disease, renal disease, an endocrinological disorder, collagen disease, neoplasm, history of excessive alcohol drinking, or acute inflammatory disease were excluded. Patients who had had an acute myocardial infarction, unstable angina, or other thromboembolism within the previous 1 month were excluded. Patients who had a severe aortic valve disease were also excluded. The final study group was limited to 323 patients. Causes of secondary hypertension were excluded as far as possible by the appropriate clinical and laboratory findings. Written informed consent was obtained from each of the 323 patients before the study. The study protocol was approved by the Ethics Committee of Yokkaichi Social Insurance Hospital.

Study protocol: Coronary angiography was performed mainly through the percutaneous brachial approach, and occasionally through the femoral approach, using the standard technique of Judkins. ${ }^{10)}$ Coronary angiography was performed after intracoronary infusion of nitroglycerin. At least 5 views of the left and 3 of the right coronary artery were obtained. Computer-assisted quantitative coronary angiography was carried out in all patients using a cardiovascular angiography analysis system. ${ }^{11,12)}$ According to the reporting system of the American Heart Association, ${ }^{13)}$ narrowing of more than $51 \%$ stenosis was considered significant.

Intraarterial blood pressure in the ascending aorta was determined using a standard fluid-filled system (5F pig-tail catheter), as we have already reported. ${ }^{14)}$ Aortic systolic pressure and aortic diastolic pressure were measured at peak systole and at end diastole, respectively. The mean pressure of the ascending aorta 
was determined electronically (Polygraph EP1600, NEC, Japan). Aortic pulse pressure was defined as aortic systolic pressure-aortic diastolic pressure.

Readings of conventional peripheral systolic pressure and diastolic pressure were taken in the right arm using a mercury-column sphygmomanometer just before the patient underwent coronary angiography. After a 10-minute rest period, supine blood pressure was measured 3 times, and the mean of the last 2 measurements was calculated. ${ }^{15)}$ Brachial systolic pressure was recorded at the first appearance of Korotkoff sounds. Brachial diastolic pressure was recorded at the disappearance of Korotkoff sounds (phase V). Brachial mean pressure was defined as $1 / 3$ brachial systolic pressure $+2 / 3$ brachial diastolic pressure. Brachial pulse pressure was defined as brachial systolic pressure minus brachial diastolic pressure.

Cardiac output was measured angiographically in each patient. Left ventricular stroke volume was estimated by measuring the left ventricular end-systolic volume using quantitative left ventricular angiography (Quantcor QCA, Siemens AG, Germany). Cardiac output was obtained by multiplying stroke volume by heart rate during the angiography.

After the patient had fasted for 12 hours, blood samples were obtained from an antecubital vein under resting conditions between 7 and 8 hours before the coronary angiography.

Each factor was determined as follows: total cholesterol (TC; automated enzymatic method), triglycerides (TG; automated enzymatic method), high-density lipoprotein cholesterol (HDL-C; selective inhibition assay), low-density lipoprotein cholesterol [LDL-C; Friedewald's equation: ${ }^{16)}$ LDL-C = TC- HDL-CTG/5], lipoprotein-(a) [Lp(a); latex immunoassay with a rabbit monoclonal antihuman Lp(a) antibody], remnant-like particle cholesterol [RLP-C; immunoadsorption assay with mouse monoclonal anti-human apolipoprotein (apo) B-100 and anti-human apo A-1 immunoaffinity mixed gels], Apo A-I, B, and E (turbidimetric immunoassays using the reagent kit, Apo Auto Daiichi, according to the manufacturer's instructions), ${ }^{17}$ ) uric acid (automated enzymatic method), blood glucose (automated enzymatic method), creatinine (alkaline picrate method), fibrinogen (thrombin time), tissue factor pathway inhibitor (TFPI; enzyme-linked immunosorbent assay), C-reactive protein (CRP; lecithin immunoassay), and serum amyloid A protein (SAA; latex agglutination nephelometric immunoassay).

The height and weight for each patient were recorded. Body mass index (BMI) was calculated as weight divided by height squared $\left(\mathrm{kg} / \mathrm{m}^{2}\right)$. All antihypertensive medicines were withdrawn at least 24 hours before the study.

Statistical analysis: Student's unpaired 2-tailed $t$ test was used for comparisons between 2 groups. Mann-Whitney's $U$ test was performed if data sets were not 
normally distributed. Differences in frequency (cross-tabulated discrete variables) were tested using the $\chi^{2}$ test. Multivariate regression analyses were performed to determine the association of coronary artery stenosis and the levels of various parameters. Correlation among parameters was tested with Pearson's correlation coefficient. Statistical analyses were performed by Stat View version 4.5 (Abacus Concepts Inc, Berkeley, CA, USA) using an Apple computer. A $P<0.05$ was considered significant in each test.

\section{RESULTS}

The mean age of the 323 patients studied was $63.4 \pm 9.3$ years (mean \pm SD) and ranged from 26 to 84 years. There were 227 males and 96 females. Coronary angiography revealed that 108 patients $(33.4 \%)$ had no significant coronary artery stenosis or occlusion and 215 patients $(66.6 \%)$ had significant organic stenosis. We defined the former 108 cases as group $\mathrm{N}$ (patients who had no stenosis) and the latter 215 cases as group $\mathrm{S}$ (patients who had at least one stenosis or occlusion). The number of patients who were found to have 1-, 2-, or 3-vessel and left main disease in the latter group was 75, 63, 58, and 19, respectively. The clinical diagnoses confirmed following coronary angiography were as follows; myocardial infarction in 99 cases, effort angina in 108 cases, angina caused by vasospasm (at rest angina) in 23 cases, silent myocardial ischemia in 20 cases, cardiac syndrome $\mathrm{X}$ in 17 cases, neurocirculatory asthenia in 35 cases, and cardiomyopathy in 21 cases.

Patient characteristics were compared between group $\mathrm{S}$ and group $\mathrm{N}$, as shown in Table I. The mean age and aortic pulse pressure were significantly higher in group $\mathrm{S}(P=0.0143$ and 0.0050 , respectively). Aortic diastolic pressure was significantly lower in group $S(P=0.0462)$. No statistically significant difference was observed between other blood pressure components and coronary artery stenosis, as can be seen in Table I.

Antihypertensive medicines may affect the blood pressure readings. Among the 323 patients studied, 176 were taking nitrates, 78 were taking $\beta$-blockers, 132 were taking angiotensin-converting enzyme (ACE) inhibitors, and 179 were taking calcium (Ca)-blockers. The percentage of subjects who took various medicines was compared between group $\mathrm{S}$ and group $\mathrm{N}$, as shown in Table II. The percentages of subjects who took nitrates, ACE inhibitors, and Ca-blockers were significantly higher in group $\mathrm{S}(P=<0.0001,<0.0001$, and 0.0224 , respectively). The lack of correlation for $\beta$-blockers may due to the small number of subjects who took the medicine.

Antihypertensive drugs may affect aortic pulse pressure measurements. We compared the value of aortic pulse pressure between patients who took the drugs 
Table I. Comparison of the Baseline Characteristics Between Patients With (Group S) and Without (Group N) Angiographically Determined Coronary Artery Stenosis

\begin{tabular}{lccc}
\hline Parameter (unit) & $\begin{array}{c}\text { Group S } \\
(n=215)\end{array}$ & $\begin{array}{c}\text { Group N } \\
(n=108)\end{array}$ & $P$ value \\
\hline Age (years) & $64.2 \pm 9.3$ & $61.4 \pm 10.7$ & 0.0143 \\
Male gender $(\%)$ & 73.2 & 63.5 & 0.0981 \\
Height $(\mathrm{cm})$ & $159.8 \pm 9.1$ & $159.4 \pm 8.6$ & 0.6929 \\
Weight $(\mathrm{kg})$ & $61.2 \pm 11.0$ & $60.8 \pm 11.3$ & 0.7831 \\
BMI $\left(\mathrm{kg} / \mathrm{m}^{2}\right)$ & $23.9 \pm 3.1$ & $23.7 \pm 3.3$ & 0.7023 \\
Cardiac output $(\mathrm{L} / \mathrm{min})$ & $5.28 \pm 1.64$ & $5.10 \pm 1.77$ & 0.3859 \\
Heart rate (beats $/ \mathrm{min})$ & $72.9 \pm 14.0$ & $71.9 \pm 15.0$ & 0.5556 \\
Aortic systolic $(\mathrm{mmHg})$ & $122.1 \pm 25.6$ & $118.0 \pm 22.4$ & 0.1672 \\
Aortic diastolic $(\mathrm{mmHg})$ & $63.2 \pm 10.4$ & $65.9 \pm 11.7$ & 0.0462 \\
Aortic mean (mmHg) & $87.3 \pm 14.6$ & $87.5 \pm 14.1$ & 0.9293 \\
Aortic PP (mmHg) & $59.0 \pm 21.5$ & $51.9 \pm 19.7$ & 0.0050 \\
Brachial systolic $(\mathrm{mmHg})$ & $119.4 \pm 15.0$ & $120.4 \pm 15.1$ & 0.5977 \\
Brachial diastolic $(\mathrm{mmHg})$ & $69.7 \pm 7.8$ & $70.4 \pm 8.7$ & 0.5055 \\
Brachial mean $(\mathrm{mmHg})$ & $86.2 \pm 8.9$ & $87.1 \pm 9.2$ & 0.4336 \\
Brachial PP (mmHg) & $50.0 \pm 12.8$ & $49.9 \pm 13.4$ & 0.9876 \\
\hline Data presented & & & \\
\hline
\end{tabular}

Data presented are mean \pm SD or percent of patients. BMI = body mass index; Aortic systolic $=$ ascending aortic systolic pressure; Aortic diastolic $=$ ascending aortic diastolic pressure; Aortic mean = ascending aortic mean pressure; Aortic PP = ascending aortic pulse pressure; Brachial systolic $=$ brachial systolic pressure; Brachial diastolic $=$ brachial diastolic pressure; Brachial mean = brachial mean pressure; Brachial PP = brachial pulse pressure.

Table II. Comparison of the Percentage of Subjects Who Took Various Medications Between Patients With (Group S) and Without (Group N) Angiographically Determined Coronary Artery Stenosis

\begin{tabular}{lcccr}
\hline Agent & $\begin{array}{c}\text { Nunber of } \\
\text { subjects }\end{array}$ & $\begin{array}{c}\text { Group S } \\
(n=215)\end{array}$ & $\begin{array}{c}\text { Group N } \\
(n=108)\end{array}$ & \multicolumn{1}{c}{$\begin{array}{c}P \text { value } \\
\left(\chi^{2}\right)\end{array}$} \\
\hline Nitrates & 176 & $67.4(n=145)$ & $28.7(n=31)$ & $<0.0001$ \\
$\beta$-blockers & 78 & $28.4(n=61)$ & $15.7(n=17)$ & 0.0673 \\
ACE inhibitors & 132 & $51.6(n=111)$ & $19.4(n=21)$ & $<0.0001$ \\
Ca-blockers & 179 & $62.8(n=135)$ & $40.7(n=44)$ & 0.0224 \\
\hline
\end{tabular}

$\mathrm{ACE}=$ angiotensin-converting enzyme.

Table III. Comparison of Aortic Pulse Pressure Between Patients Who Did Take and Did not Take Medicine

\begin{tabular}{lcccc}
\hline Agents & Number of patients & Medicine (+) & Medicine (-) & $P$ \\
\hline Nitrates & 176 & $58.9 \pm 22.6$ & $55.4 \pm 19.1$ & 0.2016 \\
$\beta$-blockers & 78 & $57.7 \pm 25.3$ & $57.5 \pm 19.5$ & 0.9409 \\
ACE inhibitors & 132 & $59.3 \pm 22.1$ & $56.4 \pm 21.0$ & 0.3013 \\
Ca-blockers & 179 & $61.4 \pm 22.3$ & $52.4 \pm 19.1$ & 0.0011 \\
\hline
\end{tabular}

Abbreviations as in Table II. 
Table IV. Simple Pearson's Correlation Coefficients Between Aortic Pulse Pressure and Baseline Characteristics

\begin{tabular}{lcc}
\hline Parameter & $\mathrm{r}$ & $P$ \\
\hline Age & 0.297 & $<0.0001$ \\
Height & -0.312 & $<0.0001$ \\
Weight & -0.207 & $<0.0001$ \\
BMI & -0.049 & 0.352 \\
Cardiac output & 0.014 & 0.8108 \\
Heart rate & -0.198 & 0.0004 \\
Aortic systolic & 0.891 & $<0.0001$ \\
Aortic diastolic & 0.087 & 0.1221 \\
Aortic mean & 0.565 & $<0.0001$ \\
Brachial systolic & 0.566 & $<0.0001$ \\
Brachial diastolic & 0.038 & 0.5467 \\
Brachial mean & 0.340 & $<0.0001$ \\
Brachial PP & 0.636 & $<0.0001$ \\
\hline
\end{tabular}

Abbreviations as in Table I.

Table V. Multivariate Statistical Analysis for Angiographically Determined Coronary Artery Stenosis With Various Parameters

\begin{tabular}{lccc}
\hline & & \multicolumn{2}{c}{$\left(\mathrm{r}^{2}=0.439\right)$} \\
Parameter (unit) & Mean value & Coefficient & $P$ value \\
\hline Age (years) & $63.2 \pm 9.8$ & -0.039 & 0.7134 \\
Aortic PP (mmHg) & $56.6 \pm 21.1$ & 0.210 & 0.0449 \\
Aortic diastolic (mmHg) & $64.1 \pm 10.9$ & -0.097 & 0.3611 \\
Uric acid (mg/dL) & $5.89 \pm 1.56$ & 0.127 & 0.2752 \\
LDL-C (mg/dL) & $116.5 \pm 30.0$ & 0.138 & 0.1859 \\
HDL-C (mg/dL) & $47.2 \pm 13.1$ & -0.089 & 0.4636 \\
TG (mg/dL) & $135.1 \pm 76.7$ & -0.062 & 0.7692 \\
Lp(a) (mg/dL) & $26.5 \pm 27.7$ & 0.055 & 0.5721 \\
RLP-C (mg/dL) & $5.16 \pm 3.23$ & 0.295 & 0.1431 \\
Blood glucose (mg/dL) & $108.1 \pm 31.9$ & 0.101 & 0.3007 \\
Creatinine (mg/dL) & $0.85 \pm 0.21$ & 0.180 & 0.1139 \\
Fibrinogen (mg/dL) & $315.7 \pm 71.9$ & 0.028 & 0.7881 \\
TFPI (ng/dL) & $23.0 \pm 6.7$ & 0.100 & 0.3658 \\
SAA $(\mu \mathrm{g} / \mathrm{mL})$ & $16.2 \pm 64.1$ & 0.190 & 0.0853 \\
\hline
\end{tabular}

LDL-C = low-density lipoprotein cholesterol; HDL-C = high-density lipoprotein cholesterol; TG = triglycerides; $\mathrm{Lp}(\mathrm{a})=$ lipoprotein-(a); RLP$\mathrm{C}=$ remnant-like particle cholesterol; TFPI = tissue factor pathway inhibitor; SAA = serum amyloid A protein. Other abbreviations as in Table I.

and those who did not (Table III). Aortic pulse pressure was significantly higher only in patients who took Ca-blockers $(P=0.0011)$. The Ca-blockers may have influenced the aortic pulse pressure values. 
The majority of population-based cohort studies have demonstrated that there is a progressive increase in pulse pressure with aging. ${ }^{5,6,18,19)}$ Therefore, we tested the correlation between the aortic pulse pressure levels and the baseline characteristics, including age (Table IV). Aortic pulse pressure showed a significant correlation with age. Multivariate regression analyses were also performed (Table V). Aortic pulse pressure was correlated with coronary artery stenosis independently of age, aortic diastolic pressure, and other variables. Each mean value of the estimated data was also described here. HbA1c was estimated in 218 cases. The mean value of $\mathrm{HbA} 1 \mathrm{c}$ was $5.61 \pm 1.12 \%$.

We examined the correlation between the number of vessels involved and aortic pulse pressure. The aortic pulse pressure levels were plotted against the number of vessels involved (Figure). Aortic pulse pressure was positively correlated with the number of vessels involved $(r=0.170, P=0.0024)$.

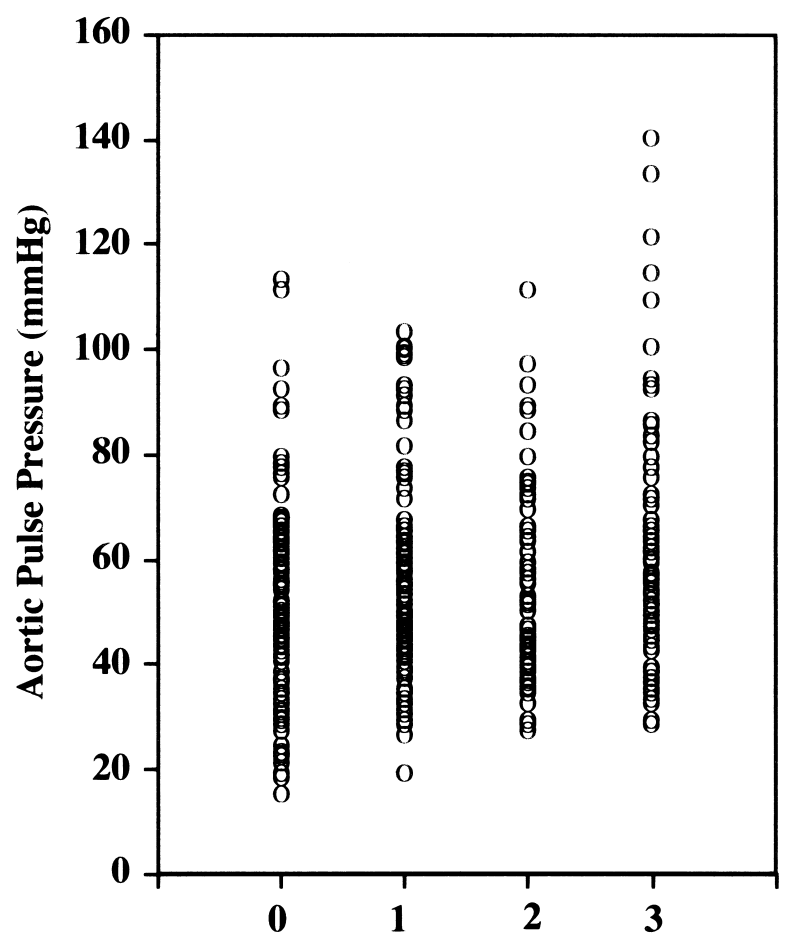

Figure. Correlation between the number of vessels involved and the level of aortic pulse pressure. The linear regression equation for the number of vessels involved: aortic pulse pressure $=52.639+3.207 \times$ numbers, $\mathrm{r}=0.170, P=$ 0.0024 . 


\section{DiSCUSSION}

The present study is the first to report that among the various components of blood pressure, central pulse pressure was significantly and independently correlated with anigiographically determined coronary artery stenosis. Moreover, we also found that central pulse pressure was positively correlated with the number of vessels involved.

Many large population epidemiological studies have shown a strong correlation between sphygmomanometrically demonstrated brachial pulse pressure and coronary heart disease. ${ }^{5-9)}$ Accordingly, it is commonly believed that arterial stiffness assumed by the brachial pulse pressure level is directly responsible for the cardiovascular complications. ${ }^{20)}$ However, this assumption may be questioned on the basis of numerous investigations that have found aortic pressure and brachial pressure differ significantly.

Whereas mean arterial pressure remains nearly constant along the arterial tree, pulse pressure increases markedly from central (aortic) to peripheral (brachial) arteries. ${ }^{23)}$ This increase is due to the propagation of the pressure wave along arterial vessels with a progressive decline in arterial diameter and an increase in arterial stiffness. The alteration modifies the summation of wave reflections at each particular point of the arterial tree, making aortic pulse pressure lower than brachial pulse pressure. ${ }^{23)}$ Using invasive techniques, Pauca, et al reported that brachial systolic pressure is $12 \mathrm{mmHg}$ higher than aortic systolic pressure, whereas aortic diastolic pressure is only 1 to $2 \mathrm{mmHg}$ lower than brachial diastolic pressure. ${ }^{21)}$ Moreover, sphygmomanometer-measured brachial diastolic pressure is substantially less accurate than systolic pressure, compared to an invasive measurement. It is important to note that it is the aortic and not the brachial arterial pressure that is "seen" by the coronary artery. ${ }^{20)}$ Central pulse pressure is assumed to be a more sensitive marker of coronary artery disease than peripheral pressure.

While the overwhelming majority of epidemiological studies have shown a relationship between arterial stiffness and coronary heart disease, ${ }^{5-9)}$ there has been little study of possible mechanisms linking arterial stiffness to coronary stenosis. The possibility that large artery, particularly aortic, stiffness may be a determinant of the vascular structure of a coronary artery is supported by some studies of coronary arteries and vascular cells in tissue culture. ${ }^{22)}$ In 1976, Nichols and O'Rourke postulated that arterial degenerative changes were due to the fatiguing effects of cyclic stress on elastin fibers and lamellae within the arterial media, and these became brittle and then fractured as a consequence of repeated cycles of stress. ${ }^{23)}$ Leung, et $a l^{24)}$ and Nakayama, et $a l^{25)}$ have confirmed that in vascular smooth muscle which was grown in culture, DNA synthesis and the rate 
of growth were greater in cells that were subjected to cyclic stretching than in cells that were grown under static conditions. These findings provide a possible explanation that cyclic stress on the vascular wall of a coronary artery may influence cellular and extracellular components of the vascular wall and may affect vascular remodelling.

There is one limitation to this study. Ca-blockers may influence pulse pressure, as can be seen in Table III. The percentage of subjects who took Ca-blockers was higher in group $\mathrm{S}$ than in group $\mathrm{N}$. We performed additional analyses among the subjects who took Ca-blockers and those who did not. In both analyses, aortic pulse pressure was significantly correlated with coronary artery stenosis $(P=$ 0.0361 , and 0.0428 , respectively) in the 179 subjects who took Ca-blockers and the 144 subjects who did not. Despite this additional analysis, we cannot rule out the possibility of the influence of Ca-blockers on pulse pressure. Our data cannot address whether a correlation between aortic pulse pressure and coronary artery stenosis might be influenced by Ca-blocker administration.

In summary, the present study has demonstrated that aortic pulse pressure was significantly and independently correlated with anigiographically determined coronary artery stenosis. Aortic pulse pressure may be one of the determinants of coronary artery stenosis. Since this is an observational, retrospective association study, the findings were not prospective and will require confirmation in a prospective study.

\section{REFERENCES}

1. Kannel WB, Dawber TR, McGee DL. Perspectives on systolic hypertension. The Framingham study. Circulation 1980; 61: 1179-82.

2. Curb JD, Borhani NO, Entwisle G, et al. Isolated systolic hypertension in 14 communities. Am J Epidemiol 1985; 121: 362-70.

3. Cambien F, Chretien JM, Ducimetiere P, Guize L, Richard JL. Is the relationship between blood pressure and cardiovascular risk dependent on body mass index? Am J Epidemiol 1985; 122: 434-42.

4. Darne B, Girerd X, Safar M, Cambien F, Guize L. Pulsatile versus steady component of blood pressure: a cross-sectional analysis and a prospective analysis on cardiovascular mortality. Hypertension 1989; 13: 392400.

5. Franklin SS. Ageing and hypertension: the assessment of blood pressure indices in predicting coronary heart disease. J Hypertens 1999; 17 (suppl 5) : S29-36.

6. Franklin SS, Larson MG, Khan SA, et al. Does the relation of blood pressure to coronary heart disease risk change with aging? The Framingham Heart Study. Circulation 2001; 103: 1245-9.

7. Mitchell GF, Moye LA, Braunwald E, et al. Sphygmomanometrically determined pulse pressure is a powerful independent predictor of recurrent events after myocardial infarction in patients with impaired left ventricular function. SAVE investigators. Survival and Ventricular Enlargement. Circulation 1997; 96: 4254-60.

8. Benetos A, Safar M, Rudnichi A, et al. Pulse pressure: a predictor of long-term cardiovascular mortality in a French male population. Hypertension 1997; 30: 1410-5.

9. Franklin SS, Kahn SA, Wong ND, Larson MG, Levy D. Is pulse pressure useful in predicting risk for coronary heart Disease? The Framingham heart study. Circulation 1999; 100: 354-60. 
10. Erren M, Reinecke H, Junker R, et al. Systemic inflammatory parameters in patients with atherosclerosis of the coronary and peripheral arteries. Arterioscler Thromb Vasc Biol 1999; 19: 2355-63.

11. Reiber JH, Serruys PW, Kooijiman CJ, et al. Assessment of short-, medium-, and long-term variations in arterial dimensions from computer-assisted quantification of coronary cineangiograms. Circulation 1985; 71: 280 8.

12. Haase J, Di Mario C, Slager CJ, et al. In-vivo validation of on-line and off-line geometric coronary measurements using insertion of stenosis phantoms in porcine coronary arteries. Cathet Cardiovasc Diagn 1992; 27: 1627.

13. Nakajima K, Saito T, Tamura A, et al. Cholesterol in remnant-like lipoproteins in human serum using monoclonal anti apo B-100 and anti apo A-1 immunoaffinity mixed gels. Clin Chim Acta 1993; 223: 53-71.

14. Masuoka H, Ito M, Kamei S, Ozaki M, Kawasaki A, Nakano T. Level of plasma tissue factor pathway inhibitor is inversely correlated with intraarterial diastolic pressure in subjects who underwent coronary angiography. Jpn Circ J 2001; 65: 165-70.

15. Benetos A, Rudnichi A, Safar M, Guize L. Pulse pressure and cardiovascular mortality in normotensive and hypertensive subjects. Hypertension 1998; 32: 560-4.

16. Friedewald WT, Levy RI, Frederickson DS. Estimation of the concentration of low-density lipoprotein cholesterol in plasma without use of the preparative ultracentrifuge. Clin Chem 1972; 18: 499-502.

17. Ikeda T, Shibuya Y, Senba U, et al. Automated immunoturbidimetric analysis of six plasma apolipoproteins : correlation with radial immunodiffusion assays. J Clin Lab Anal 1991; 5: 90-5.

18. Franklin SS, Gustin W4th, Wong ND, et al. Hemodynamic patterns of age-related changes in blood pressure. The Framingham Heart Study. Circulation 1997; 96: 308-15.

19. Chae CU, Pfeffer MA, Glynn RJ, Mitchell GF, Taylor JO, Hennekens CH. Increased pulse pressure and risk of heart failure in the elderly. JAMA 1999; 281: 634-9.

20. Safar ME, Blacher J, Pannier B, et al. Central pulse pressure and mortality in end-stage renal disease. Hypertension 2002; 39: 735-8.

21. Pauca AL, Wallenhaupt SL, Kon ND, Tucker WY. Does radial artery pressure accurately reflect aortic pressure? Chest 1992; 102: 1193-8.

22. Baumbach GL. Is pulse pressure a stimulus for altered vascular structure in chronic hypertension? Hypertension 1991; 18: 728-9.

23. Nichols WW, O'Rourke MF. McDonald's blood flow in arteries. 4th ed. London, UK: Arnold, Hodder Headline Group; 1998: 54-113, 170-222, 284-93, 347-401.

24. Leung DY, Glagov S, Mathews MB. Cyclic stretching stimulates synthesis of matrix components by arterial smooth muscle cells in vitro. Science 1976; 191: 475-7.

25. Nakayama K, Suzuki S, Sugi H. Physiological and ultrastructural studies on the mechanism of stretch-induced contractile activation in rabbit cerebral artery smooth muscle. Jpn J Physiol 1986; 36: 745-60. 